\title{
A Rotation Invariant Face Recognition Method Based on Complex Network
}

\author{
Wesley Nunes Gonçalves, Jonathan de Andrade Silva, \\ and Odemir Martinez Bruno \\ Instituto de Física de São Carlos \\ University of São Paulo \\ Av. do Trabalhador Sãocarlense, 400, São Carlos, São Paulo, Brazil \\ wnunes@ursa.ifsc.usp.br, jsilva@icmc.usp.br, bruno@ifsc.usp.br
}

\begin{abstract}
Face recognition is an important field that has received a lot of attention from computer vision community, with diverse set of applications in industry and science. This paper introduces a novel graph based method for face recognition which is rotation invariant. The main idea of the approach is to model the face image into a graph and use complex network methodology to extract a feature vector. We present the novel methodology and the experiments comparing it with four important and state of art algorithms. The results demonstrated that the proposed method has more positive results than the previous ones.
\end{abstract}

Keywords: Face Recognition, Complex Network, Graph.

\section{Introduction}

Face recognition is a field of investigation that has been become popular in the science and also in the industry. It has gained increasing attention in the community due to the successful applications in various areas, specially in the security industry. Although face recognition is not the most accurate biometric method, it is the most non invasive technology. This characteristic makes it suitable for many special secure applications, such as crowd supervision in airports.

In the literature, there are two main approaches to facial feature extraction: analytic and holistic approaches. The former extracts information based on structural face regions (e.g. eyes, mouth and nose), which contain important information for human identification. The latter considers the whole face image in the identification process. Holistic approach has some advantages to the analytic approach, such as: (i) it uses information of texture and shape which contribute to face recognition and (ii) it does not require pre-segmentation methods. For both approaches, most of the methods are not rotation invariant. This causes inaccuracy in real applications as it is very common for people to incline their head. In this work, we considered the second approach and propose a novel methodology based on complex network which are rotation invariant.

Complex network is a relatively recent field of research that combines graph analysis and statistical physics. Complex network research is close to graph research. In fact, the distinction between them is the approach. On one hand,

I. Bloch and R.M. Cesar, Jr. (Eds.): CIARP 2010, LNCS 6419, pp. 426-433. 2010.

(C) Springer-Verlag Berlin Heidelberg 2010 
graphs are quite traditional in computer science and mathematics. On the other hand, complex networks emerge from physics and use a statistical approach to perform the analysis. Complex networks have been popularized with works of Watts and Strogatz [1] and Barabasi [2], and have been applied in different fields of science, such as physics, biology, economy among others. Despite the successful applications, there are few applications in computer vision and pattern recognition. Some applications, such as texture classification [3] and pixel clustering [4, can be found in the literature.

This paper introduces a novel method for face recognition which is based on the complex network theory. Using the holistic approach, a face image is transformed into multi-scale graphs and then, they are analyzed using the complex network theory. In the analysis, measurements are extracted in order to compose a feature vector that characterizes the face image. To assess the quality of the proposed method, we conducted two experiments. The first experiment was performed using face images from a public database, while the second experiment test the proposed method by classifying rotate face images. For comparisons, we selected four popular face recognition methods belonging to holistic approach.

The paper is organized as follows. Section 2 and 3 briefly review the complex networks and their measurements, respectively. In Section 4, the proposed method is described in detail for face recognition applications. The experiments performed and the results are presented in Section 5. Finally, the conclusions and improvement of the method are discussed in Section 6.

\section{Complex Networks}

Complex network is a recent area which joins graph theory and statistical physics analysis. The complex networks are represented by graphs consisting of a set of vertices connected by edges. The graph is described by $G(V, E)$, where $V=$ $\left\{v_{1}, v_{2}, \ldots, v_{n}\right\}$ is the set of vertices and $E=\left\{e_{1}, e_{2}, \ldots, e_{M}\right\}$ is the set of edges.

The complex networks were classified according to their properties into three main models: random networks, small-world and scale-free. Random networks, proposed by Erdós e Rényi [5], are considered the simplest model. In this model, the edges are added at random. Watts and Strogatz, who studied random networks in applications, concluded that networks have highly connected vertices with closed paths of length three. Therefore, a complex network that has no edges added completely at random, called small-world network, was proposed 1]. Barabási and Albert proposed a scale invariant network namely scale-free network 2. In this model, the distribution of connectivity of vertices follows a scale-free distribution given by $P(k) \sim k^{-\gamma}$, with $\gamma=3$ and $n \rightarrow \infty$ [2].

\section{$2.1 \quad$ Measures}

Once a system is modeled by a complex network, measures can be extracted in order to analysis and characterize the network topology. A survey where the reader can find a good discussion about different measurements is available in 
6]. In this work, a network is used to model a face image and then, measures are extracted for the pattern recognition proposal. The sections below describe measures related to connectivity and hierarchy, both presenting a good performance in the topology characterization [76].

Connectivity. Complex networks present as main characteristic a set of vertices and edges that forms particular topographic properties. These properties can be measured by the number of connections among vertices [7. For weighted networks, the vertex degree $k w_{i}$ is obtained by $k w_{i}=\sum_{j=1}^{N} w_{i j}$, where $w_{i j}$ is the weight of the connection between vertex $i$ and $j$. Although simple, the vertex grade performs well in characterizing the networks, as it provides information about the topology and distribution of the connectivity throughout the network.

Hierarchical Degree. Hierarchy performs an important role in the complex network approach. The hierarchical level can be defined as a graph dilatation $\delta(g)$, resulting in a sub graph containing the vertices from $g$ and the vertices directly connected to $g$. The graph dilation can be iterative. The $d$-dilatation process, which means that, a graph $g$ is dilated $d$ times, is shown below:

$$
\delta_{d}(g)=\underbrace{\delta(\delta(\ldots(g) \ldots))}_{d}
$$

The $d$-ring of a subgraph $g$ is defined as $R_{d}(g)$ and it is composed by the vertices and edges according to:

$$
R_{d}(g)=V\left(\delta_{d}(g)\right)-V\left(\delta_{d-1}(g)\right)
$$

where $V($.$) is the set of vertices and - is the subtraction operation between sets.$

The hierarchical degree $k_{i}^{d}$ can be defined by the number of edges that connect the rings $R_{d}(i)$ and $R_{d-1}(i)$ to the vertex $i$. The weighed hierarchical degree $k w_{i}^{d}$ is calculated similarly by summing the weights.

\section{Face Recognition Using Complex Networks}

The proposed method can be split into two stages: network modeling and feature extraction. To model an image as a network $G=(V, E)$, each pixel is mapped to a vertex of the set $V$. Two vertices $i$ and $j$, related to the pixels $p_{i}$ and $p_{j}$, are connected if the distance between the pixels is less than a given radius $r$. The connection weight is defined by the pixel intensity difference (see Equation 3).

$$
w_{i j}= \begin{cases}\left.\mid I\left(p_{i}\right)-I\left(p_{j}\right)\right) \mid & \text { if } \operatorname{dist}\left(p_{i}, p_{j}\right) \leq r \\ 0, & \text { otherwise }\end{cases}
$$

where $w_{i j}$ is the edge weight, $I(x)$ is the pixel intensity of the pixel $x$, dist is the Euclidean distance and $r$ is the radius of neighborhood.

The next step is to transform the graph into a complex network, with a rich structure and topology. In this work, the authors demonstrated that a simple 
regular graph similar to the one obtained until now can be transformed into a small world complex network. The transform, defined as $\psi(t, G)$, consists of the selection of edges according to their weights and a threshold $t$, which can vary from $0 \leq t \leq \max (W)$ (see Equation 4). Figure 1 illustrates the transform $\psi(t, G)$.

$$
w_{i j}= \begin{cases}w_{i j}, & \text { if } w_{i j} \leq t \\ 0, & \text { otherwise }\end{cases}
$$

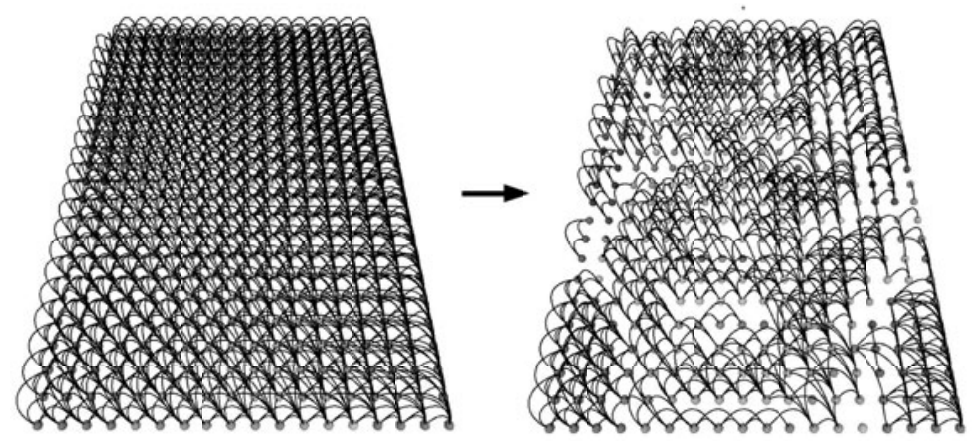

Fig. 1. Transformation $\psi$ in a complex network

The transform $\psi(t, G)$ can be considered as a multi-scale network analysis. For each value of $t$ the original network is transformed into a $t$ scaled network. Thus, the $t$ scaled network presents different properties and reveals the structure and topology related to its scale. For small values of $t$, the network provides information of image details, presenting better small sets of pixels or regions and as $t$ increases, it presents better global information. For high values of $t$, the network can provide the image edges. The proposal of our method is to combine measures from some values of $t$ achieving a multi-scale analysis.

\subsection{Feature Vector Extraction}

The face characterization is performed by extracting measures from networks $G_{t}=\psi(t, G)$ varying the thresholding $t$. The values are between the interval $t_{0} \leq t \leq t_{f}$ and incremented by a constant $t_{\text {inc }}$. The metrics discussed in Section 2.1 are calculated for each vertex of $G_{t}$. Then, a vector $\boldsymbol{f}_{t}$ is carried out by calculating the mean, variance and kurtosis from each metric. If $n$ metrics are used, the feature vector $f_{t}$ for a given scale $t$ is a $3 n$ vector (mean variance and kurtosis for each metric). Equation 5 shows how to calculate $\boldsymbol{f}_{t}$, where $i$ is related to each metric. The final feature vector $f$ for a given face is the concatenation of the individual feature vectors $f_{t}$ at different scales, according to Equation 6.

$$
\begin{aligned}
& m_{i}=\left[\operatorname{mean}\left(G_{t}\right) \text { variance }\left(G_{t}\right) \text { kurtosis }\left(G_{t}\right)\right] \\
& \boldsymbol{f}_{t}=\left[\begin{array}{lllll}
m_{0} & \ldots & m_{i} & \ldots & m_{n}
\end{array}\right]
\end{aligned}
$$




$$
\boldsymbol{f}=\left[f_{t_{0}} f_{\left(t_{0}+t_{\text {inc }}\right)} \ldots f_{t_{f}}\right]
$$

\section{Face Recognition Experiments}

In order to validate the proposed method, a set of experiments was carried out using a well-known public face database - ORL database 8 . This database has 10 images of 40 subjects, with changes in illumination, facial details and expressions. Two experiments were conducted: parameter analysis and comparison with other methods. For both experiments, 10 -folds cross-validation was carried out using K-neighbor nearest [9], with $k=1$. A simple statistical classifier was chosen in order to show evidence of the feature role in pattern recognition.

\subsection{Analysis of the Method Parameters}

This subsection presents a study of the proposed method parameters and its consequences on the pattern recognition performance. The parameters considered are: (i) the radius influence for the transformation which converts the image to the network, (ii) the thresholds used for the multi-scale transform, (iii) the measures considered to compose the feature vector. The objective of this analysis is to present the method behavior and determine the best set of parameters.

The main parameters of the method are the neighbor radius $r$ and the initial threshold $t_{0}$. Both parameters are related to the network structure and changes in them can directly influence the face recognition performance. The parameter analysis starts choosing a range for $r$ and $t_{0}$ where the method achieves its optimum performance. For this, we evaluated various combinations of these parameters and the method achieved the best performance for $r \approx \sqrt{73}$ and $t_{0} \approx 10$. These values can be combined with the other parameters to find the optimal parameter set of the method.

Figure 2 presents an analysis of all the parameters. In Figure 2(a) the plot ratio $r$ versus classification rates is shown. Notice that the classification rate achieves its maximum $98 \%$ for $r=\sqrt{73}$. Figure 2(b) shows a plot of $t_{0}$ versus classification rates. The maximum classification rate is achieved for $t_{0}=10$. For low values of $t_{0}$, such as those less than 5 , the network can be highly disturbed by noise. On the other hand, for high values of $t_{0}$, the network cannot present the face details and lose important face information. Figures 2(c) and 2(d) present respectively $t_{i n c}$ versus classification rates and $t_{f}$ versus classification rates. To compute the plots $t_{f}=t_{0}+x * t_{\text {inc }}$ was used, where $x$ is the number of times of $t_{\text {inc }}$. This function makes $t_{i n c}$ and $t_{f}$ correlated values. In the first analysis, $x=7$ (see Figure 2(d)) was used. The plot shows that the best results are achieved for high values of $t_{i n c}$. It is expected as, for high values of $t_{i n c}$ the method can explore a better range of scales for the multi-scale analysis. In Figure 2(d), the plot shows the classification rates versus the number of thresholds, given by $x$ which determines $t_{f}$. Notice that the curve achieves its maximum when $x=7$ and it maintains stabilized. Using these four plots, we can determine the best chosen parameters, which are $r=\sqrt{73}, t_{0}=10, t_{\text {inc }}=10$ and $t_{f}=t_{0}+7 * t_{\text {inc }}$. 

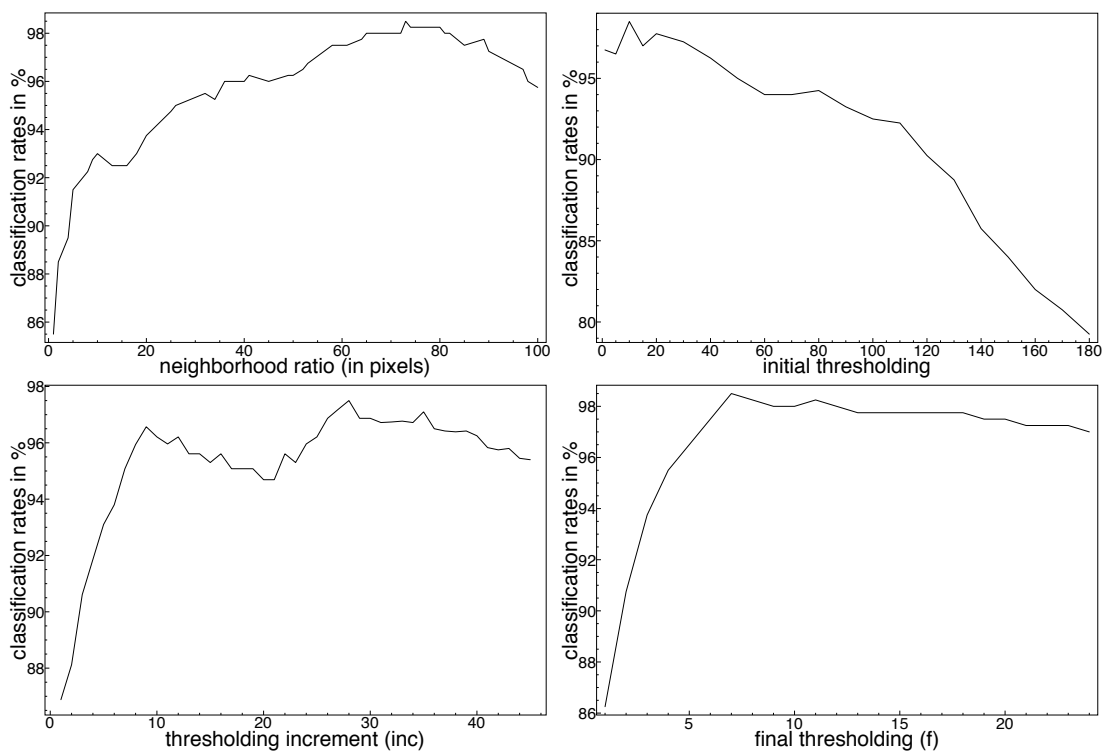

Fig. 2. (a) Comparison results for different radius, (b) Threshold $t_{0} \mathrm{X}$ Correct percent, (c) Threshold $t_{\text {inc }} \mathrm{X}$ Correct percent and (d) Threshold $t_{f} \mathrm{X}$ Correct percent

The feature vector consists of metrics extracted from the transformed networks. Three measurements were evaluated: the degree, two level hierarchical degree and three level hierarchical degree. Table 1 compares performance of the three combined feature vector (Full) to each individual metric in terms of classification rate, the false acceptance rate (FAR) and false rejection rate (FRR). The best individual metric was the degree which achieved a correct percentage of $94.75 \%$. The combined feature vector (full) has the best correct percentage and the smallest FRR and FAR, which suggests that the individual metric extracts different information from the networks.

Table 1. Comparison results for measures and the three combined feature vector (Full)

\begin{tabular}{|c|c|c|c|}
\hline & Correct Percentage & FAR & FRR \\
\hline \hline Full & $98.5 \%$ & 0.00045 & 0.015 \\
Degree & $94.75 \%$ & 0.00145 & 0.053 \\
Hier. D. 2 & $93.75 \%$ & 0.00178 & 0.063 \\
Hier. D. 3 & $91.5 \%$ & 0.00233 & 0.085 \\
\hline
\end{tabular}

\subsection{Comparing with Other Methods}

The results of the proposed method are compared to the results of the eigenfaces [10], fisherfaces [11, laplacianfaces [12] and neighborhood preserving embedding - NPE 13 in Table 2. It is important to notice that various combinations of 
parameters and numbers of descriptors were carried out for all methods. Only the best result achieved was considered in the comparison. The proposed method proved to be better than the others in the classification rates and also in the FAR and FRR.

Table 2. Results of the proposed method and state of the art methods

\begin{tabular}{|c|c|c|c|}
\hline & Correct Percentage & FAR & FRR \\
\hline \hline Proposed Method & $98.5 \%$ & 0.0005 & 0.015 \\
FisherFaces & $95.73 \%$ & 0.0011 & 0.042 \\
NPE & $94.75 \%$ & 0.0014 & 0.053 \\
EigenFaces & $94.55 \%$ & 0.0014 & 0.0545 \\
LaplacianFaces & $94.25 \%$ & 0.0014 & 0.0575 \\
\hline
\end{tabular}

The performance of most face recognition algorithms are influenced by the position of the face in the image. Usually, the faces must be carefully aligned to extract the features relative to a fixed coordinate system. In order to evaluate the rotation invariance of the proposed method, each image from the ORL database was rotated in four directions $\left(-60^{\circ},-30^{\circ}, 30^{\circ}, 60^{\circ}\right)$, totaling 2000 images.

The proposed method results in the increased database are compared with other methods in Table 3 . In this experiment, the cross-validation strategy with 2 -folds was performed to increase the amount of images in the test step $(50 \%$ of the images are used to test the model in the 2 -folds cross-validation). The proposed method had more positive results than the others in the classification rates and also in the FAR and FRR. The method achieved a correct percentage of $93.65 \%$ compared to only $58.45 \%$ achieved by the Fisherfaces.

Table 3. Results of the methods in the increased ORL database

\begin{tabular}{|c|c|c|c|}
\hline & Correct Percentage & FAR & FRR \\
\hline \hline Proposed Method & $93.65 \%$ & 0.0019 & 0.064 \\
FisherFaces & $58.45 \%$ & 0.0168 & 0.415 \\
LaplacianFaces & $54.45 \%$ & 0.0202 & 0.456 \\
EigenFaces & $42.75 \%$ & 0.0197 & 0.573 \\
NPE & $41.8 \%$ & 0.0171 & 0.582 \\
\hline
\end{tabular}

\section{Conclusion}

This paper presented a novel approach to face recognition based on the complex network theory. In the proposed method, the face image is mapped onto a regular graph and then it is transformed into a small-world network. Using the complex network theory, various features are extracted, providing a feature vector that can identify faces. 
The method was compared with popular and the state-of-art methods. The proposed method proved to be better than the others in the classification rates and also in the FAR and FRR measures. Moreover, according to the nature of the graphs, the proposed method is rotation invariant, which differs from most of the holistic face recognition methods. As part of the future work, we plan to focus on evaluating the proposed method in large scale face image data sets.

Acknowledgements. The authors acknowledges support from CNPq.

\section{References}

1. Watts, D.J., Strogatz, S.H.: Collective dynamics of 'small-world' networks. Nature 393(6684), 440-442 (1998)

2. Barabasi, A.L., Albert, R.: Emergence of scaling in random networks. Science 286(5439), 509-512 (1999)

3. Backes, A.R., Casanova, D., Bruno, O.M.: A complex network-based approach for boundary shape analysis. Pattern Recognition 42(1), 54-67 (2009)

4. Silva, T.C., Zhao, L.: Pixel clustering by using complex network community detection technique. In: ISDA, pp. 925-932. IEEE Computer Society, Los Alamitos (2007)

5. Erdős, P., Rényi, A.: On random graphs. I. Publ. Math. Debrecen 6, 290-297 (1959)

6. Costa, L.F., Rodrigues, F.A., Travieso, G., Boas, P.R.V.: Characterization of complex networks: A survey of measurements. Advances In Physics 56, 167-242 (2007)

7. Boccaletti, S., Latora, V., Moreno, Y., Chavez, M., Hwang, D.U.: Complex networks: Structure and dynamics. Physics Reports 424(4-5), 175-308 (2006)

8. Samaria, F.S., Harter, A.C.: Parameterisation of a stochastic model for human face identification. In: IEEE Workshop on Appl. of Computer Vision, pp. 138-142 (1994)

9. Mitchell, T.M.: Machine Learning. McGraw-Hill, New York (1997)

10. Belhumeur, P.N., Jo, a.P.H., Kriegman, D.J.: Eigenfaces vs. fisherfaces: Recognition using class specific linear projection. IEEE PAMI 19(7), 711-720 (1997)

11. Zuo, W., Wang, K., Zhang, D., Zhang, H.: Combination of two novel lda-based methods for face recognition. Neurocomputing 70(4-6), 735-742 (2007)

12. He, X., Yan, S., Hu, Y., Niyogi, P., Zhang, H.J.: Face recognition using laplacianfaces. IEEE Trans. Pattern Anal. Mach. Intell. 27(3), 328-340 (2005)

13. He, X., Cai, D., Yan, S., Zhang, H.J.: Neighborhood preserving embedding. In: IEEE International Conference on Computer Vision, vol. 2, pp. 1208-1213 (2005) 